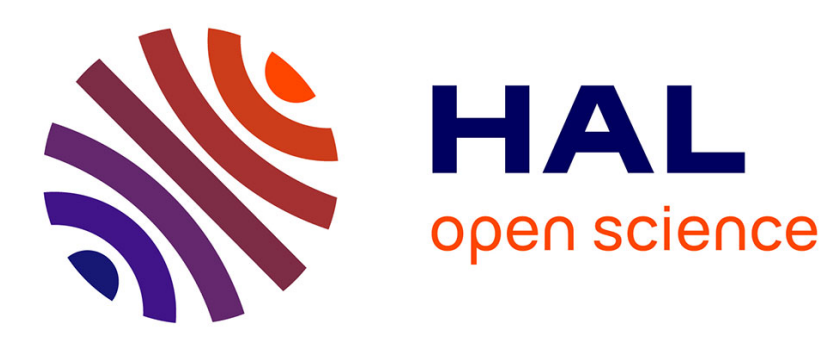

\title{
La difficile articulation entre les espaces du quotidien chez les enfants sans logement
}

\author{
Emmanuelle Guyavarch, Erwan Le Méner, Nicolas Oppenchaim
}

\section{To cite this version:}

Emmanuelle Guyavarch, Erwan Le Méner, Nicolas Oppenchaim. La difficile articulation entre les espaces du quotidien chez les enfants sans logement. Les Annales de la Recherche Urbaine, 2016, 111, pp.18-30. hal-01329601

\section{HAL Id: hal-01329601 https://hal.science/hal-01329601}

Submitted on 10 Jun 2016

HAL is a multi-disciplinary open access archive for the deposit and dissemination of scientific research documents, whether they are published or not. The documents may come from teaching and research institutions in France or abroad, or from public or private research centers.
L'archive ouverte pluridisciplinaire $\mathbf{H A L}$, est destinée au dépôt et à la diffusion de documents scientifiques de niveau recherche, publiés ou non, émanant des établissements d'enseignement et de recherche français ou étrangers, des laboratoires publics ou privés. 


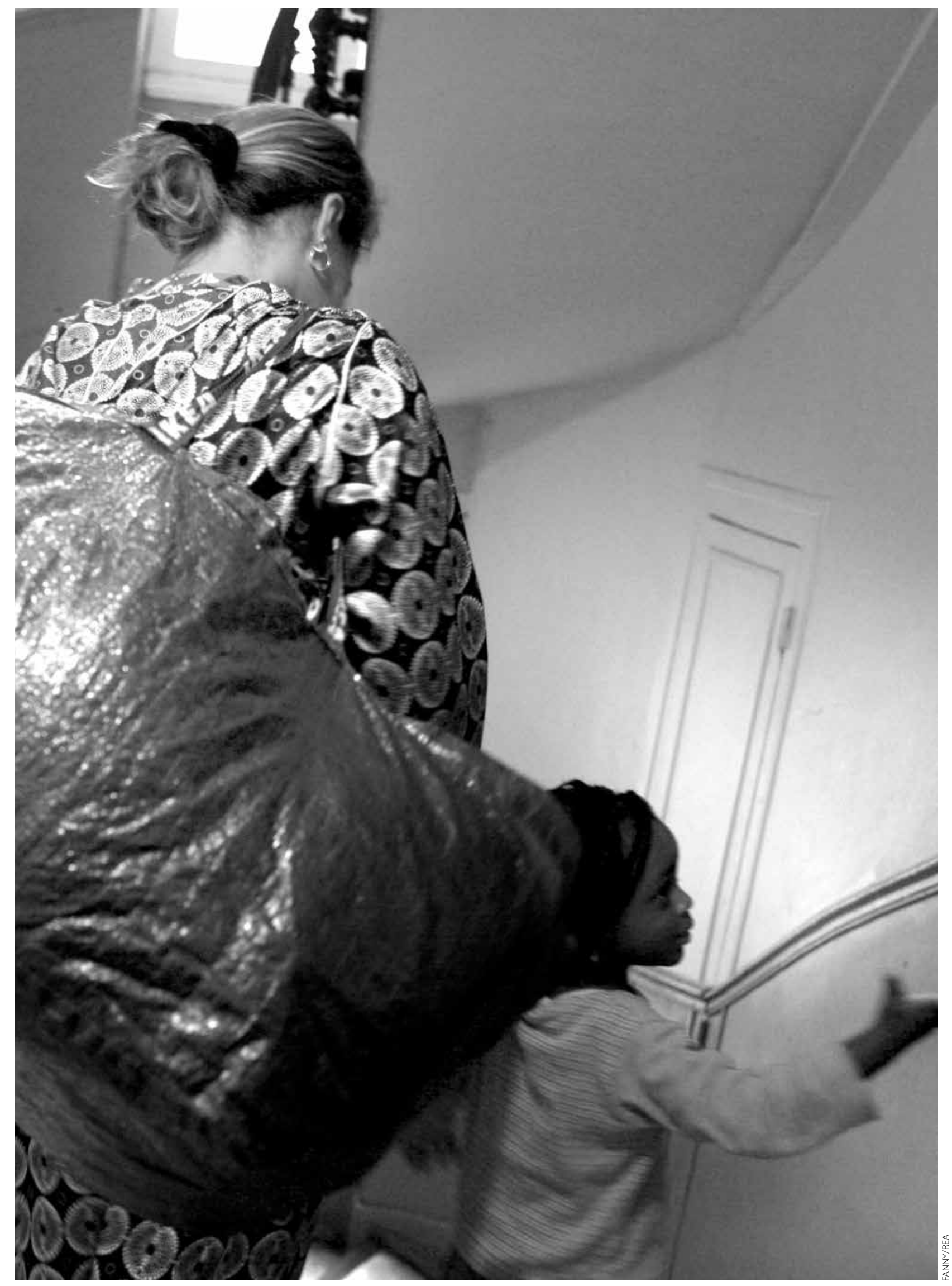




\section{La difficile articulation entre les espaces du quotidien chez les enfants sans logement}

Emmanuelle Guyavarch, Erwan Le Méner, Nicolas Oppenchaim

Les sans-domicile sont de plus en plus nombreux : 141500 en 2012 en France métropolitaine, soit une augmentation de $50 \%$ par rapport à 2001 (Yacouncq et al., 2013). Le phénomène est principalement urbain : plus des trois quarts d'entre eux, dont 30000 enfants mineurs, résident dans une commune de plus de 20000 habitants. Un nombre croissant de ménages avec enfants (appelés «familles » dans la suite de l'article, selon le vocable en usage dans le monde de l'intervention sociale) occupe le système d'hébergement (Guyavarch et Le Méner, 2014), en particulier dans d'importantes agglomérations où l'accueil généraliste a complété ou suppléé les carences des dispositifs spécialisés dans l'accueil d'étrangers, de demandeurs d'asile en particulier (Dourlens, 2006 ; Frigoli, 2004 ; Noblet, 2001).

Ainsi, depuis la fin des années 1990, un nombre croissant de familles, majoritairement étrangères, est privé de logement et hébergé par le 115 de Paris (Guyavarch et Le Méner, 2014). Début 2013 en Île-de-France, plus de 10000 familles habitent en hôtel social ${ }^{1}$, en centre d'hébergement d'urgence (CHU), de réinsertion sociale

1. L'appellation « hôtel social » désigne ici plusieurs catégories d'établissements qui diffèrent par leur équipement : des hôtels meublés, des établissements de tourisme, des résidences hôtelières à vocation sociale. Le mode d'hébergement en hôtel peut varier sensiblement d'un opérateur et d'un département à l'autre. Alors que les organisations parisiennes privilégient un accueil à durée indéterminée, les 115 et les conseils généraux des départements voisins ont plutôt tendance à fixer une limite de durée au séjour des familles, parfois assortie de déménagements forcés d'un établissement à l'autre. D'autre part, certains CHU et CHRS se sont spécialisés totalement ou partiellement dans l'accueil de familles. L'hébergement est généralement collectif, mais il existe aussi des appartements dispersés autour du siège de la structure. Les CHU proposent en principe un hébergement pour une durée plus brève que les CHRS, et sont globalement moins bien équipés. Enfin, les centres d'accueil des demandeurs d'asile (Cada), offrent aux demandeurs d'asile des prestations et équipements voisins de ceux des CHRS.
(CHRS) ou en centre d'accueil pour demandeurs d'asile (Cada). En font partie 17662 enfants de moins de treize ans, dont 5894 âgés de six à douze ans, c'est-à-dire scolarisables dans le primaire (Guyavarch et al., 2014). Pourtant, les travaux français sur le sans-abrisme ne se sont guère intéressés aux familles, et encore moins aux enfants qui les composent ${ }^{2}$. Cette invisibilité académique redouble celle qui continue de s'appliquer plus largement à la pauvreté des enfants (Cazottes, 2013 ; Stettinger, 2014). Cet article entend alors contribuer à l'étude des formes de pauvreté infantile, à partir d'une enquête sur les familles sans domicile ${ }^{3}$. Quelles sont les conditions de vie des enfants sans logement? Quelles sont les conséquences de l'absence de domicile fixe sur les pratiques enfantines des espaces quotidiens?

En suivant au long cours et en interrogeant directement des enfants, certains contextes caractéristiques du système d'hébergementsontapparus, compliquantconsidérablement leur vie quotidienne. Par exemple, en hôtel social, les enfants tendent à être traités comme n'importe quel adulte vis-à-vis du règlement de l'établissement par le personnel et indirectement par les organisations orientant leurs familles; cette situation entraîne des limitations imposées aux

2. Aucune des plus de cinq cents références en français de sciences sociales recensées entre 1987 et 2012 sur le sans-abrisme (Choppin et Gardella, 2012) n'aborde directement le sujet.

3. Nous parlons tantôt de familles sans domicile ou sans logement. Les deux expressions désignent le fait d'être hébergé par l'assistance faute de logement propre. La première renvoie plutôt à une appellation utilisée dans les enquêtes statistiques (et au mode de définition et de comptage utilisé dans ces enquêtes) ; la seconde met l'accent sur le logement comme problème central pour les familles hébergées et comme horizon de l'action publique.

Les Annales de la recherche urbaine $\mathrm{n}^{\circ} 111$, 2016, pp. 18-29, (C)MEDDE, Puca. 
usages de l'hébergement, pour les parents comme pour les enfants : il est généralement interdit de garder des affaires trop volumineuses dans sa chambre, de la décorer ou d'y accrocher des posters, d'y inviter des amis et de jouer dans les couloirs... (Le Méner, 2015). Il a également été montré que l'instabilité résidentielle qui caractérise l'hébergement d'urgence entraîne des difficultés pour se rendre à l'école (Le Méner et Oppenchaim, 2015a). Cet article cherche tout d'abord à compléter le tableau des difficultés rencontrées par ces enfants dans les espaces du quotidien, en s'intéressant à la fois à l'hébergement et ses alentours et aux mobilités en dehors du quartier. Comment des enfants, qui ont souvent en commun l'expérience de fréquents déménagements, parviennent-ils ou non à s'approprier l'environnement de leur lieu d'habitation? Plus largement, quels usages de la ville leurs mobilités permettent-elles de retracer ? La première partie présente les enquêtes sur lesquelles s'appuie notre analyse ; la seconde tente ensuite de caractériser le contexte de pauvreté dans lequel vivent ces enfants, en s'intéressant aux ressources de leur famille, mais aussi à leurs conditions d'hébergement. La pauvreté de ces enfants peut en effet être traitée sous deux angles : celui de la faiblesse des ressources matérielles, et celui d'une forte vulnérabilité résidentielle. Cette vulnérabilité renvoie aussi bien à des conditions d'hébergement dégradées qu'à une importante instabilité résidentielle, touchant plus particulièrement les enfants hébergés en hôtel et ceux pour qui l'absence de logement est relativement récente. À cet égard, l'usage des espaces quotidiens diffère-t-il chez les enfants, selon leur degré de vulnérabilité résidentielle? Cette question fera l'objet de la dernière partie, principalement analysée à partir d'un indicateur particulier de vulnérabilité, le nombre de déménagements au cours des douze derniers mois.

\section{Un ensemble d'enquêtes sur les enfants et les familles sans logement}

Cet article est issu du projet Enfants et familles sans logement (Enfams), conduit à l'Observatoire du Samusocial de Paris depuis 2011. L'objectif général de ce projet de sciences sociales et d'épidémiologie est de décrire les conditions de vie et les trajectoires des familles sans logement. Il s'appuie notamment sur la réalisation de plusieurs enquêtes qualitatives : sur le système d'hébergement, sur la vie de résidents en hôtels, sur l'accessibilité et le recours aux soins en périnatalité. Cependant, cet article se fonde à titre principal sur une

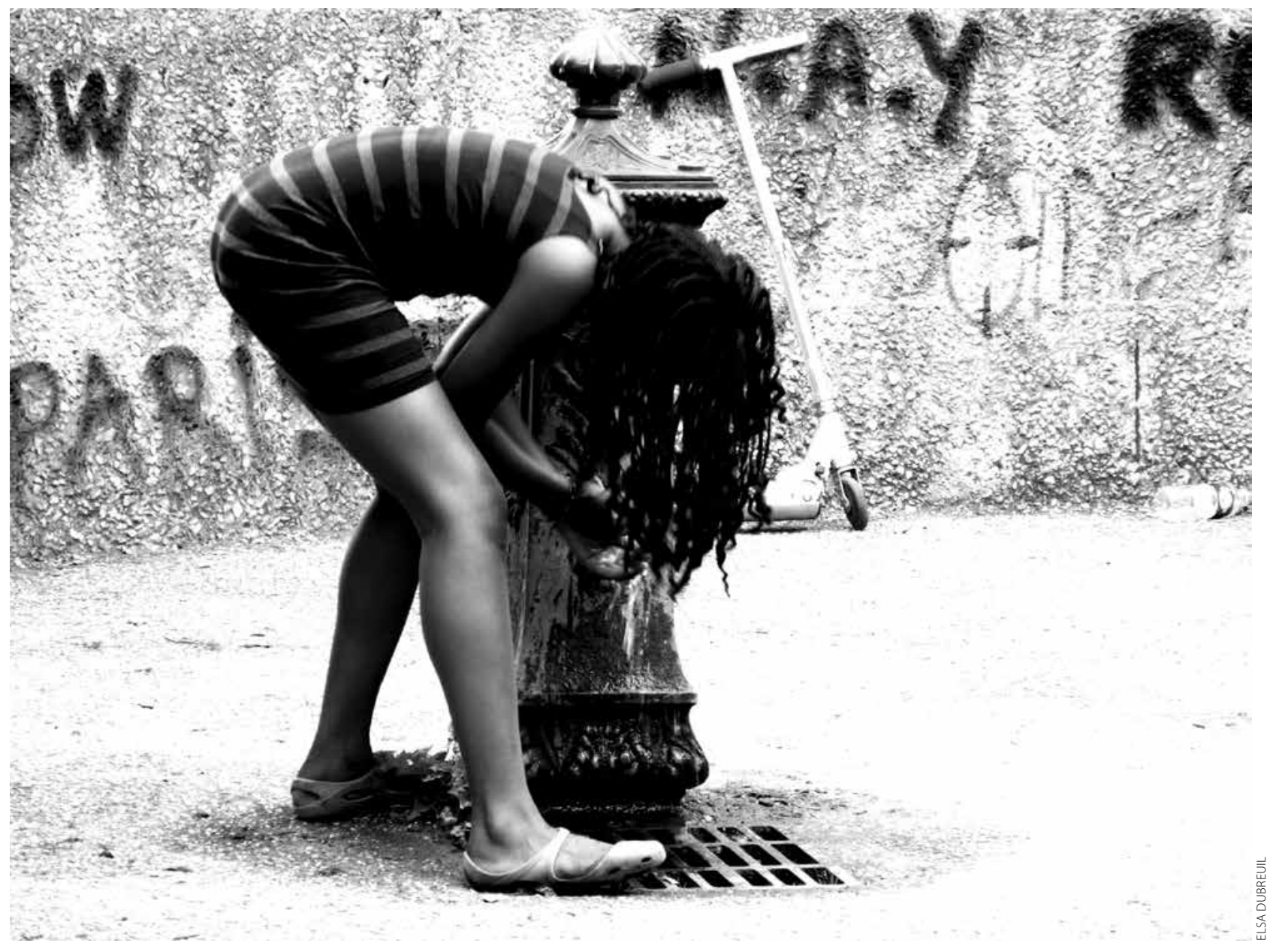


enquête quantitative qui vise à estimer la taille de la population sans logement en famille en Île-de-France, menée au premier semestre 2013 auprès d'un échantillon aléatoire de 801 familles hébergées dans les établissements précités (hôtels sociaux, CHU, CHRS, Cada). Ces structures proposent en principe un accueil de courte durée, l'enquête exclut donc les ménages sans-abri au sens strict, installés dans des campements par exemple, mais aussi des ménages bénéficiant de formes plus durables d'hébergement, en résidence sociale ou en logement transitoire. Les familles du panel, tirées au sort, devaient comporter au moins un enfant de moins de treize ans, et les parents parler une des dix-sept langues de l'étude. Au sein de chaque ménage, un adulte et un enfant ont été enquêtés. Le questionnaire adressé au parent établissait les caractéristiques sociodémographiques du ménage, s'intéressait à la trajectoire migratoire et d'hébergement du parent interrogé, portait sur les conditions de vie, la situation administrative, les revenus ou encore l'état de santé des membres du ménage. Chez les enfants, deux classes d'âge ont été distinguées : entre la naissance et 5 ans révolus, et entre 6 et 12 ans révolus. Nous n'avons pas interrogé d'enfants de plus de 12 ans, afin d'éviter de mener des investigations sur de trop petits effectifs, ceux-ci étant moins nombreux que leurs cadets. Les 232 enfants en âge d'être scolarisés en primaire ont répondu à un questionnaire d'une trentaine de minutes portant sur la vie et les relations sociales à l'hôtel et à l'école, sur leurs pratiques culturelles et leurs sociabilités hors de l'école. L'information collectée a été complétée par un questionnaire d'une demi-heure soumis aux parents sur leur enfant, insistant notamment sur ses mobilités et ses pratiques scolaires et extrascolaires ${ }^{4}$.

\section{Les caractéristiques de la pauvreté des enfants sans logement}

La pauvreté des enfants sans logement (de six à douze ans) peut être abordée sous trois angles complémentaires : en premier lieu, d'après les ressources de leurs familles, qui expriment un dénuement généralisé ; puis en s'intéressant aux conditions d'habitation, qui ne sont pas sans incidence sur les usages enfantins de l'espace domestique et de l'hébergement ; enfin, en considérant l'instabilité résidentielle des enfants. En effet, si presque tous les enfants ont en commun de vivre dans des ménages pauvres et des habitations confinées, ils ne présentent pas tous un parcours résidentiel aussi heurté, marqué par de nombreux déménagements, d'une structure d'hébergement à une autre.

4. Une présentation plus détaillée de la méthodologie se trouve dans le rapport des premiers résultats (Guyavarch et al., 2014).

\section{Des enfants de familles pauvres}

Début 2013, en Île-de-France, au moins 5894 enfants âgés de 6 à 12 ans vivent sans logement. La plupart résident à Paris (19\%), en Seine-Saint-Denis (26\%) et dans le Val-de-Marne (18\%), départements les mieux dotés en structures d'accueil. Ces enfants vivent dans des ménages dont le revenu moyen déclaré est de 315 euros par unité de consommation. Il apparaît que $93 \%$ des familles disposent d'un revenu mensuel par unité de consommation inférieur pour moitié au seuil de pauvreté, qui est de 814 euros en 2011, souvent dans des foyers monoparentaux (44\%) et des familles avec de nombreux enfants ( $21 \%$ avec trois enfants au moins).

Comme pour les enfants pauvres franciliens en général (Chemineau et Flamand, 2014), les parents des enfants de notre enquête se trouvent dans une situation professionnelle délicate. Seuls $27 \%$ des parents déclarent travailler, avec ou sans contrat de travail, alors que $58 \%$ ne travaillent pas et ne touchent ni RSA ni allocation chômage. Or, chez les enfants sans logement, le travail s'avère protecteur : le revenu moyen par unité de consommation est de 543 euros par mois parmi les enfants dont au moins un parent travaille, même à temps partiel, contre 178 euros pour ceux dont aucun parent ne travaille. Cette pauvreté monétaire généralisée, plus intense lorsque les parents ne travaillent pas, varie sensiblement en fonction de la situation administrative des parents : les enfants de sans-papiers ou de demandeurs d'asile (interdits de travail légal, sauf dans de rares conditions) sont plus pauvres que les enfants dont les parents ont un titre de séjour permettant de travailler. Les ressources des familles sont alors complétées par des dons. On estime par exemple que $55 \%$ des foyers des enfants de 6 à 12 ans ont bénéficié, au cours du mois précédent l'enquête, de dons de nourriture de la part d'une association, $16 \%$ d'un particulier. Ces dons concernent davantage les familles qui ne déclarent aucune ressource issue du travail ou aucune allocation.

\section{Un habitat pauvre mais inégal}

Un autre trait caractéristique de la pauvreté des familles sans logement renvoie à leur habitation. L'occupation des hôtels ou des centres collectifs d'hébergement déroge à toutes les normes en vigueur concernant le logement. La situation normale dans ces établissements correspond à celle d'un 


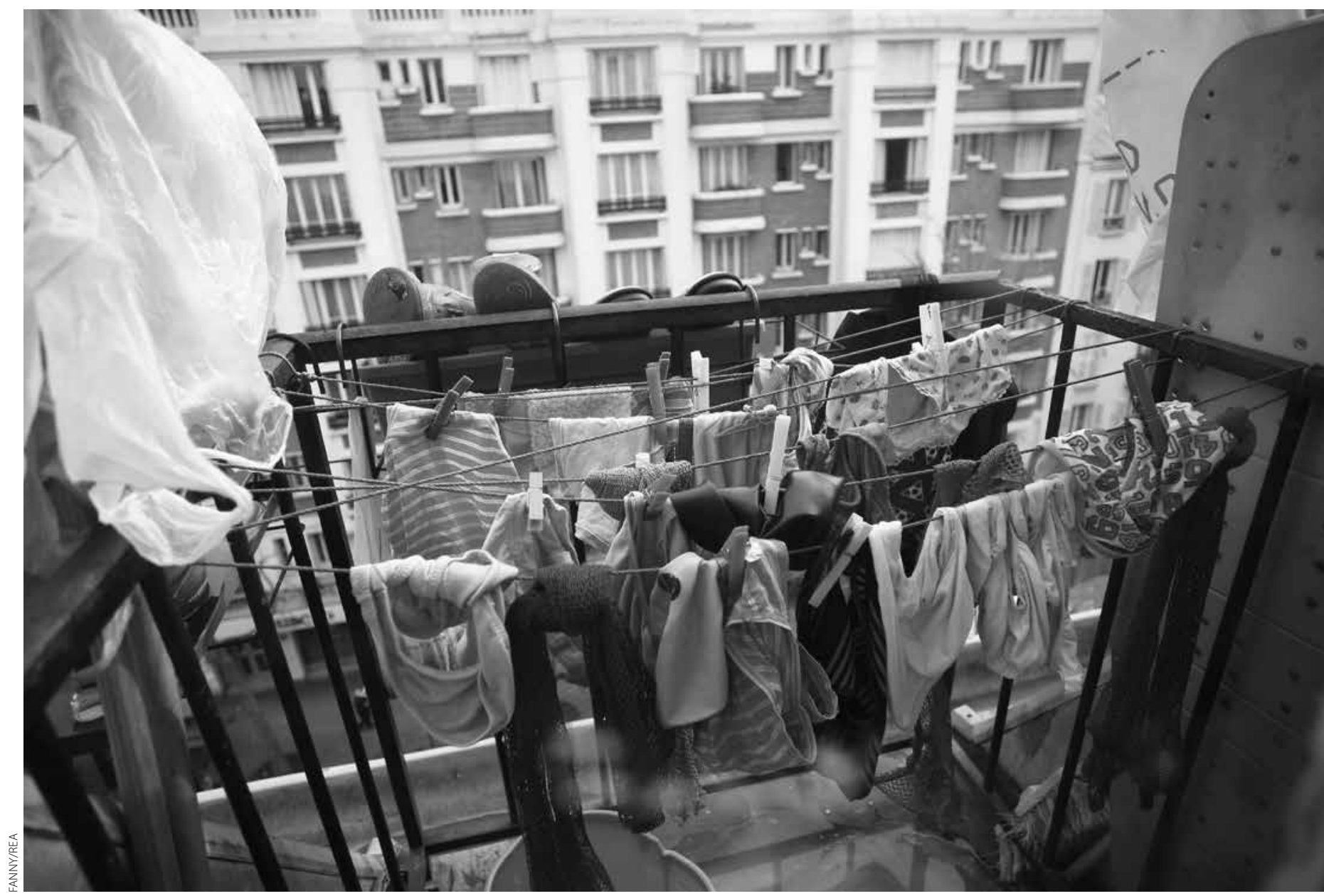

surpeuplement « accentué ${ }^{5}$ » toutefois, certaines structures proposent un hébergement en appartement. D’autre part, la taille des pièces ne dit pas tout de leur habitabilité. En hôtel social par exemple, la configuration des chambres, leur ameublement, leur équipement en général, entrent pleinement en ligne de compte dans l'évaluation qu'en font les parents et les enfants (Le Méner, 2015). Si les conditions d'habitat diffèrent selon les établissements, celles-là influencent grandement les pratiques quotidiennes des enfants dans leur lieu de résidence. Elles impliquent par exemple très fréquemment un confinement dans la chambre en hôtel social. Pour des enfants, a fortiori lorsque l'épisode sans-domicile s'allonge, habiter un espace réduit

5. Une chambre d'hôtel de sept mètres carrés peut accueillir au plus une personne, une chambre de neuf mètres carrés au plus deux personnes, une chambre de treize mètres carrés au plus trois personnes, et ainsi de suite. À titre de comparaison et d'après l'Enquête Logement 2006, en Île-de-France, les enfants pauvres mais logés disposent en moyenne de dix-sept mètres carrés habitables au sein de leur foyer ; ils n'occupent pas moins dans huit cas sur dix un logement surpeuplé (Labrador, 2011). Sur la définition du surpeuplement accentué, voir Insee, [en ligne] www.insee.fr/fr/ methodes/default.asp?page=definitions/indice-peuplement-insee.htm. peut être durablement préjudiciable à leur développement (Buckner, 2008), mais cela influence également sans doute leur rapport aux espaces extérieurs.

De façon peu surprenante compte tenu du fonctionnement du système d'hébergement (Le Méner, 2013), le mode principal d'hébergement est l'hôtel social. Les enfants y résident dans plus de sept cas sur dix. Vient ensuite l'hébergement en CHRS (20\%), en Cada (5\%) et en CHU (2\%). L'hébergement proposé y est globalement frustre. Concernant les équipements habituellement situés à l'intérieur du logement, répondant à des besoins élémentaires et de l'ordre du quotidien, plus d'un enfant sur cinq ne dispose pas de toilettes dans son hébergement; près d'un enfant sur dix occupe une pièce sans douche, et environ la même proportion est contrainte d'utiliser une douche qui ne fonctionne pas toujours ou sans eau chaude. En Île-de-France, moins de $2 \%$ des logements peuvent être considérés comme "sans confort ", c'est-àdire sans eau courante, WC ou sanitaire (Charrier et al., 2009). Par ailleurs, les frères et sœurs ont chacun leur lit dans seulement $45 \%$ des familles sans logement, tandis que plus de trois enfants sur dix dorment avec un de leur parent, plus particulièrement en hôtel où cette situation concerne la moitié d'entre eux. Pour les enfants qui 
vont à l'école primaire, l'absence de bureau ou de table pour faire ses devoirs est également courante $(44 \%)$. Néanmoins, seule une minorité d'enfants se plaint de la promiscuité dans laquelle ils doivent travailler : si $68 \%$ souhaiteraient avoir plus de place pour faire leurs devoirs, seuls $41 \%$ disent être gênés par la présence d'une autre personne, de bruits, ou divers dérangements, au moment de faire leurs exercices chez eux.

Toutefois, des différences sont observables entre les types d'établissement. En hôtel, chaque famille dispose d'une ou de deux chambres, selon sa taille. Dans les autres types d'établissement, l'accueil en collectivité côtoie l'hébergement diffus dans des appartements, où vivent un ou plusieurs ménages. En tenant compte de ces différences, les appartements paraissent nettement mieux équipés que l'ensemble des autres structures en ce qui concerne les sanitaires et les douches. Parmi les hébergements en collectivité, les Cada présentent le pire niveau d'équipement, le CHRS le meilleur (excepté pour l'accès à Internet), tandis que les hôtels se trouvent dans une situation intermédiaire.

Étant donné leurs conditions d'hébergement, nous avons demandé aux enfants ce qu'ils souhaiteraient changer dans leur chambre ou dans leur structure. Ils témoignent d'aspirations qui contrastent nettement avec la situation actuelle de leur foyer : les enfants aimeraient en priorité avoir "plus de place ", une chambre pour eux, et un espace de jeu dans leur habitation. Ces analyses prennent tout leur sens au regard des usages effectifs de l'espace de l'hébergement. Les chambres sont petites et ne permettent pas d'y faire forcément tout ce que l'on souhaiterait, quand on le souhaiterait ; et dans la majorité des établissements, en hôtel en particulier, l'espace collectif ne se prête guère à une utilisation ludique. Il est interdit de faire du bruit dans les couloirs, de jouer en général dans les parties communes, parfois d'utiliser tout simplement les espaces verts qui entourent l'établissement, bien que des tolérances existent ici ou là (Le Méner, 2015).

Face à la pauvreté de l'habitation et aux usages limités de l'établissement, certaines activités sont-elles réalisées en contrepartie aux alentours de l'hébergement? Pourrait-on même repérer une appropriation d'autant plus forte du quartier que les enfants sont confinés à l'intérieur de leur structure ? Pour répondre à ces questions, il importe également de prendre en compte la vulnérabilité résidentielle des enfants sans logement, considérée sous l'angle de la fréquence de leurs déménagements.

\section{L'instabilité résidentielle des enfants}

Le système d'hébergement s'est développé comme une solution provisoire aux problèmes de logement des familles, eux-mêmes relatifs à des statuts administratifs précaires. Lautonomisation du secteur de l'hébergement et du suivi social et administratif des familles migrantes a renforcé une logique de mise à l'abri, typique de l'urgence sociale (Le Méner et Oppenchaim, 2012). Dans cette perspective, l'hébergement répond au besoin immédiat d'avoir un toit. Il est ainsi adossé à une conception minimale de l'habiter, largement indifférente à l'espace occupé (que cet espace désigne l'établissement lui-même, son environnement, ou ses relations avec les espaces du quotidien des parents ou des enfants, l'école en premier lieu). Face à l'augmentation continue des demandes obligeant les hébergeurs à travailler en flux tendu, l'hébergement induit une forte instabilité résidentielle des familles : puisque l'objectif est uniquement de les mettre à l'abri, en cas de suroccupation du logement liée à une naissance, d'indisponibilité temporaire de chambres dans l'hôtel, ou de conflits avec l'hôtelier, les familles peuvent être transférées dans un autre hôtel, dont la destination n'est pas prédéterminée et sur laquelle elles ont très peu de prise. Les « réorientations » sont effectuées en fonction des places disponibles; rien ne garantit que le prochain hôtel se trouvera à proximité du précédent, du lieu de leur suivi social ou encore des établissements scolaires de leurs enfants. On discerne une dimension de contrainte dans la plupart des mobilités résidentielles : si elle n'est pas nécessairement propre aux ménages les plus fragiles (Authier, 2010), elle paraît accentuée chez les familles pauvres - plus du quart des déménagements de familles pauvres franciliennes n'aurait pas lieu par choix (Labrador, 2011).

Dans le cas des familles sans logement, la non-maitrise de la mobilité résidentielle semble tout simplement la règle, et ces familles déménagent davantage que la population générale. Alors qu'en moyenne, une personne sur dix déménage chaque année en France (Donzeau et Pan Khé Shon, 2009), les familles franciliennes sans logement changent environ en moyenne trois fois d'hébergement par an (Guyavarch et al., 2014). Cette moyenne considérable cache d'importantes disparités : quatre familles sur dix n'ont pas changé d'hébergement durant les douze derniers mois, $22 \%$ d'entre elles ont déménagé une fois et $38 \%$ plus d'une fois. Ces disparités sont fortement liées à la durée de présence dans le système d'hébergement. Durant la première année sans logement, le nombre moyen de déménagement est maximal, mais il décroît ensuite au fur et à mesure des années. Ainsi, $78 \%$ des familles ont déménagé au moins une fois durant leur première année dans le système d'hébergement, alors que moins d'une sur deux déménage durant les années suivantes. Les familles sans logement depuis un an révolu auront déménagé en moyenne un peu moins de quatre fois par an, celles sans logement depuis deux ou trois ans révolus auront déménagé près de deux fois par an, tandis que les familles sans logement depuis au moins quatre ans auront déménagé une fois par an.

Un indicateur particulier permet d'apprécier l'effet de la mobilité résidentielle sur l'appropriation des espaces 
quotidiens des enfants : le nombre de déménagements durant les douze derniers mois. Cet indicateur semble préférable à celui du nombre moyen de déménagements par an, qui exclut de facto les familles sans logement depuis moins d'un an, soit plus d'un quart des familles. Or, l'instabilité est particulièrement forte en début de parcours d'hébergement, période qui correspond souvent à une arrivée récente en France et à un apprentissage des systèmes institutionnels, notamment scolaire. L'influence de l'instabilité résidentielle sur les pratiques des enfants est ainsi souvent le résultat conjoint du caractère déstabilisant des déménagements et d'une moindre connaissance des dispositifs institutionnels, liés à une arrivée récente en France et dans le système d'hébergement. L'arrivée récente en France influence ainsi le rapport des enfants à leur hébergement, mais aussi à certaines de leurs pratiques extrascolaires, par exemple les départs en vacances.

\section{Le poids de la vulnérabilité résidentielle sur I'appropriation des espaces du quotidien}

L'analyse du rapport à l'école des enfants révèle des difficultés accrues par l'instabilité résidentielle, aussi bien pour être scolarisé que pour suivre normalement les cours (Le Méner et Oppenchaim, 2015b). Cependant, au prix d'efforts importants (notamment des journées et des déplacements trop longs), l'école constitue un point d'ancrage essentiel pour la plupart des enfants, comme d'ailleurs des parents (Le Méner et Oppenchaim, 2015a). Peut-on observer un semblable et paradoxal engagement dans d'autres espaces du quotidien ? Les sociabilités et les pratiques des enfants au sein de leur hébergement, dans leur quartier et à l'extérieur de celui-ci, paraissent souvent différenciées selon le type d'établissement, mais surtout limitées par l'instabilité résidentielle. Ces deux variables ne sont d'ailleurs pas indépendantes : l'instabilité résidentielle est très marquée en hôtel et en $\mathrm{CHU}$, bien moindre chez les enfants vivant en Cada et surtout en CHRS.

\section{Des sociabilités limitées dans le lieu de résidence}

Pour les enfants, l'établissement dans lequel ils sont hébergés n'est pas une maison comme une autre. Ils semblent conscients d'y vivre pour une durée limitée, d'y manquer de place et notamment d'espaces pour jouer. À la conception du foyer comme d'un chez soi paraît se substituer l'importance de préserver un " entre nous ", peu dépendant du lieu matériel de l'hébergement (Le Méner, 2015), mais essentiel face à la vulnérabilité résidentielle (Kirkman et al., 2010). Cependant, les plaintes et les critiques que les parents ou les enfants peuvent formuler à l'égard de leur hébergement ne recouvrent pas forcément l'éventail réel et la diversité des pratiques quotidiennes qui s'y font jour. Pour certains enfants par exemple, un centre d'hébergement d'urgence peut représenter une halte salutaire dans un parcours résidentiel chaotique, où l'on se fait des amis et où l'on découvre certains plaisirs et un sentiment de sécurité (Mougin, 2009). Qu'en est-il pour les enfants de notre enquête?

Plusieurs items des questionnaires interrogent les relations sociales des enfants à l'intérieur de leur hébergement. Un premier élément de cadrage doit être apporté : certains établissements interdisent les visites. De fait, près de huit enfants sur dix n'ont reçu aucune visite dans l'année écoulée, notamment parmi ceux hébergés en hôtel : seuls 14 \% d'entre eux ont pu recevoir des enfants extérieurs à la structure durant cette période. Néanmoins, les familles peuvent recevoir des voisins dans leur chambre. Mais d'après les parents, seulement $39 \%$ des enfants se sont amusés dans leur chambre avec d'autres enfants au cours du mois précédent l'enquête. Ceux qui vivent en Cada (60 \%) et en CHRS (46\%) jouent le plus avec leurs pairs. Par contraste, c'est en hôtel que l'on compte la part la plus importante d'enfants ne jouant jamais avec d'autres enfants dans leur chambre : un peu plus de sept enfants sur dix sont dans ce cas. Ces différences pourraient renvoyer à l'importance de l'intervention sociale voire éducative au sein des Cada et CHRS, favorisant les relations entre pairs, absente en revanche au quotidien dans les hôtels sociaux. À l'inverse, en hôtel, les enfants font communément l'expérience d'un enfermement relatif aux nombreuses règles et interdictions qui encadrent leurs usages aussi bien des parties collectives que des chambres (Le Méner, 2015). De fait, pour les enfants vivant en hôtel, le lieu d'hébergement n'est que rarement un endroit où l'on voit ses copains, qu'ils vivent ou non sur place : seuls $23 \%$ de ces enfants disent y recevoir des copains (contre $56 \%$ et $45 \%$ pour les enfants vivant respectivement en Cada en CHRS).

Cependant, les différences de règlements internes et les degrés d'intervention sociale variables selon les établissements ne sont pas seuls en cause ; l'instabilité résidentielle entre aussi en compte. Les enfants ayant déménagé dans l'année reçoivent ainsi significativement moins de visites de copains dans leur chambre : d'après les parents, $47 \%$ des enfants n'ayant pas déménagé au cours de l'année écoulée jouent avec d'autres enfants dans leur chambre ; ils ne sont que trois sur dix parmi ceux qui ont déménagé. En outre, parmi les enfants vivant en hôtel, seule la moitié des enfants ayant déménagé au cours de l'année passée disent avoir des copains dans l'hôtel où ils vivent, contre $70 \%$ chez les enfants n'ayant pas changé d'établissement. D'ailleurs, dans les hôtels, les enfants jouent assez peu dans les chambres de leurs voisins (moins d'un tiers des répondants).

De plus, les amitiés sont d'autant plus résistantes que les enfants changent peu d'établissement. Dans l'ensemble, un peu plus d'un quart des enfants revoient des copains partis de leur hébergement, mais cette valeur passe à un tiers chez les enfants n'ayant pas déménagé dans l'année. Cette possibilité de garder le contact 


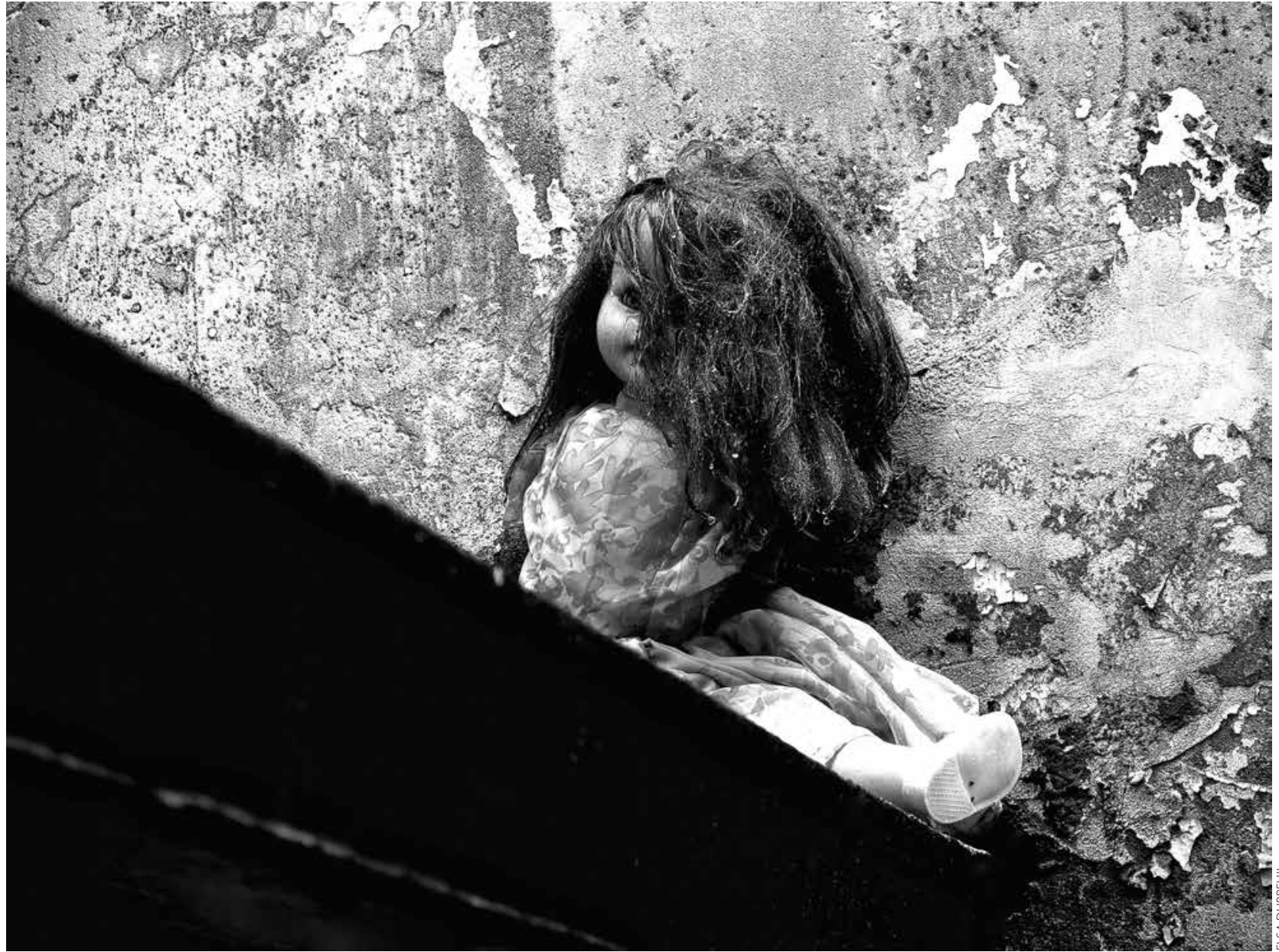

dépend aussi très fortement du lieu d'hébergement des enfants : ce sont ceux hébergés en Cada, et surtout en CHRS, qui revoient encore des copains ayant déménagé. Trois éléments peuvent expliquer cette variation selon les structures d'hébergement : d'une part, la plus grande stabilité résidentielle en CHRS et en Cada permet aux enfants de nouer des liens plus durables entre camarades; d'autre part, les enfants disposent de plus de facilités dans ces structures pour accueillir chez eux leurs anciens camarades; enfin, il est plus facile de garder le contact avec des camarades lorsque ceux-ci déménagent vers un hébergement stable, ce qui est sans doute davantage le cas pour les anciens résidents de Cada ou de CHRS que pour les enfants vivant en CHU ou en hôtel.

Ce double effet du type d'hébergement et de l'instabilité résidentielle se retrouve dans l'analyse d'autres moments de sociabilité, comme les anniversaires. C'est ainsi en CHRS que les enfants fêtent le plus souvent leur anniversaire $(54 \%)$. D'autre part, plus on déménage, moins on fête l'anniversaire de l'enfant, notamment à l'intérieur de la structure d'hébergement : $51 \%$ des enfants n'ayant pas déménagé dans l'année ont fêté leur anniversaire dans la structure d'hébergement, $36 \%$ parmi ceux qui ont déménagé une fois, $28 \%$ parmi les enfants qui ont déménagé plus d'une fois. Alors qu'un encadrement éducatif pourrait favoriser la tenue de ces moments sortant de l'ordinaire, de fréquents déménagements ne permettent pas aux familles d'organiser ces événements. À l'inverse, seul l'effet de l'instabilité résidentielle se retrouve dans l'analyse des relations des enfants avec les adultes de l'établissement, habitants ou membres du personnel. Les enfants les plus instables sont moins nombreux à déclarer connaître les adultes de la structure. Les plus stables ont pour leur part davantage tendance que les autres enfants à apprécier l'ensemble du personnel de l'établissement.

Au total, les sociabilités et les relations sociales des enfants sans logement paraissent tout à fait limitées, à l'intérieur de leur hébergement. Toutefois, il importe de nuancer cette analyse en fonction du type d'hébergement occupé et de la mobilité résidentielle des enfants. L'hébergement en CHRS et en Cada, c'est-à-dire dans des centres globalement mieux équipés et encadrés que les autres, paraît plus hospitalier qu'en hôtel, a fortiori qu'en $\mathrm{CHU}$, où les familles ne font que passer. Le sentiment d'enfermement ressenti par les enfants vivant en hôtel rendrait ainsi bien compte du fait que trois enfants sur quatre en hôtel souhaitent en déménager. Mais l'instabilité résidentielle paraît là encore très corrosive : les enfants les 
plus instables, peu importe où ils résident, sont ceux qui souhaitent le moins déménager, alors même qu'ils sont aussi nombreux à déclarer ne pas aimer du tout l'endroit où ils résident. N'oublions cependant pas que l'instabilité résidentielle est la plus forte au début de la trajectoire dans le système d'hébergement, qui correspond bien souvent à une arrivée récente en France. Sur ce point, la date d'arrivée des parents en France a une influence beaucoup plus significative que l'instabilité résidentielle sur la volonté de déménager : $55 \%$ des enfants dont les parents sont arrivés il y a moins de deux ans souhaiteraient déménager, contre $79 \%$ des enfants dont les parents sont arrivés il y a plus de deux ans. Cette liaison statistique semble indiquer une usure des enfants au fur et à mesure de leur parcours dans le système d'hébergement.

\section{Une appropriation variable du quartier}

Étant donné les contraintes pesant sur les sociabilités résidentielles des enfants hébergés, ceux-ci n’investissentils pas davantage et en contrepartie l'environnement de leur hébergement ? Cette question paraît avoir d'autant plus d'importance que les parents estiment majoritairement que le quartier où ils résident présente une "situation générale meilleure que celle d'autres quartiers » (six cas sur dix), et constitue un «bon endroit pour élever des enfants » (sept cas sur dix).

Nous nous sommes intéressés aux liens amicaux dans le quartier : d'après leurs parents, $62 \%$ des enfants rencontreraient des amis dans le quartier. Ces valeurs sont moindres pour les enfants en hôtel et en CHU. Elles décroissent surtout avec l'instabilité résidentielle : alors que trois quarts des enfants n'ayant pas déménagé rencontrent des amis dans leur quartier, ce n'est le cas que de la moitié de ceux qui ont déménagé une fois et de $39 \%$ de ceux qui ont déménagé plus d’une fois. En matière de sociabilité amicale, on peut se demander s'il existe d'une part une continuité entre la sociabilité qui s'exerce dans la structure d'hébergement et celle qui se pratique dans ses environs, et d'autre part un impact général négatif de la mobilité résidentielle. C'est ce que donnent également à entendre les enfants interrogés.

L'instabilité résidentielle paraît en effet plus déterminante que le lieu d'hébergement sur les sociabilités extrascolaires des enfants. Ce sont les enfants les plus stables (au niveau résidentiel) qui déclarent ainsi s'amuser le plus souvent entre pairs en dehors de l'école : $56 \%$ de ceux qui n'ont pas déménagé durant l'année sont dans ce cas, contre $38 \%$ de ceux qui ont déménagé une fois, et $43 \%$ de ceux qui ont déménagé plus fréquemment encore. Les enfants les plus stables sont aussi ceux qui vont le plus souvent chez des copains ne vivant pas dans leur établissement, ou qui les voient en dehors de l'école. Ces enfants auraient ainsi des relations sociales plus intégrées dans leur quartier. De même, les sociabilités qui ont lieu en dehors du domicile des enfants, « dehors », dans « un parc », ou plus précisément « un parc du quartier », concernent également davantage les enfants stables sur le plan résidentiel : trois quarts des enfants n'ayant pas déménagé dans l'année voient par exemple des copains "dehors ", alors que seule la moitié de ceux ayant déménagé sont dans ce cas.

D'autres indicateurs de l'appropriation des environs de l'hébergement ont été mesurés (se promener dans le quartier, utiliser des équipements publics pour des activités ludiques, sportives ou culturelles, faire partie d'une association). Dans tous les cas, l'instabilité résidentielle différencie les pratiques. À titre d'illustration, $82 \%$ des enfants qui n'ont pas déménagé durant les douze derniers mois se promènent dans le quartier, contre $75 \%$ de ceux qui ont déménagé au moins une fois ; $82 \%$ de ceux qui n’ont pas déménagé utilisent les équipements du quartier, contre $70 \%$ de ceux qui ont déménagé ; $36 \%$ des enfants n'ayant pas déménagé fréquentent une association du quartier, contre $25 \%$ de ceux qui ont déménagé une fois et $15 \%$ de ceux qui ont déménagé plus d'une fois (notons par ailleurs que les enfants qui ne fréquentent pas d'association dans le quartier n'en fréquentent pas non plus en dehors).

Il n'est donc guère étonnant que les enfants les plus stables déclarent apprécier leur quartier : $76 \%$ des enfants n'ayant pas déménagé durant les douze derniers mois disent bien aimer leur quartier, contre $60 \%$ des enfants ayant déménagé au moins une fois. À l'inverse, seuls $10 \%$ déclarent ne pas aimer du tout leur quartier, contre $24 \%$ des enfants ayant déménagé. Cette appréciation différenciée du quartier pourrait être la conséquence d'une moins bonne connaissance des équipements urbains de proximité. Ainsi, $55 \%$ des enfants n'ayant pas déménagé estiment qu'il y a assez de parcs dans leur quartier, contre $35 \%$ de ceux qui ont déménagé au moins une fois. Si l'on fait l'hypothèse que le nombre de parcs à proximité du domicile est relativement similaire quel que soit le quartier de résidence des enfants sans logement, cette appréciation différenciée du quartier pourrait s'expliquer par une moindre exploration des équipements urbains de proximité. À l'inverse, cette appréciation différenciée n'est pas liée à la perception de l'insécurité dans le quartier, car les enfants stables au niveau résidentiel ne s'y sentent pas plus en sécurité. Ils sont même plus nombreux que les autres à éviter certains endroits, comme si une meilleure connaissance contribuait à une appréciation négative de certains lieux.

Ce moindre investissement du quartier par les enfants ayant déménagé durant l'année ne s'explique néanmoins pas seulement par la moins bonne connaissance des équipements urbains de proximité. Elle est aussi fortement liée aux déplacements quotidiens pour se rendre à l'école, qui limitent le temps disponible des enfants, les plus instables au niveau résidentiel étant fréquemment scolarisés en dehors de leur commune de résidence. Ce dernier facteur contribue également à limiter les activités extrascolaires des enfants en dehors de leur quartier. 


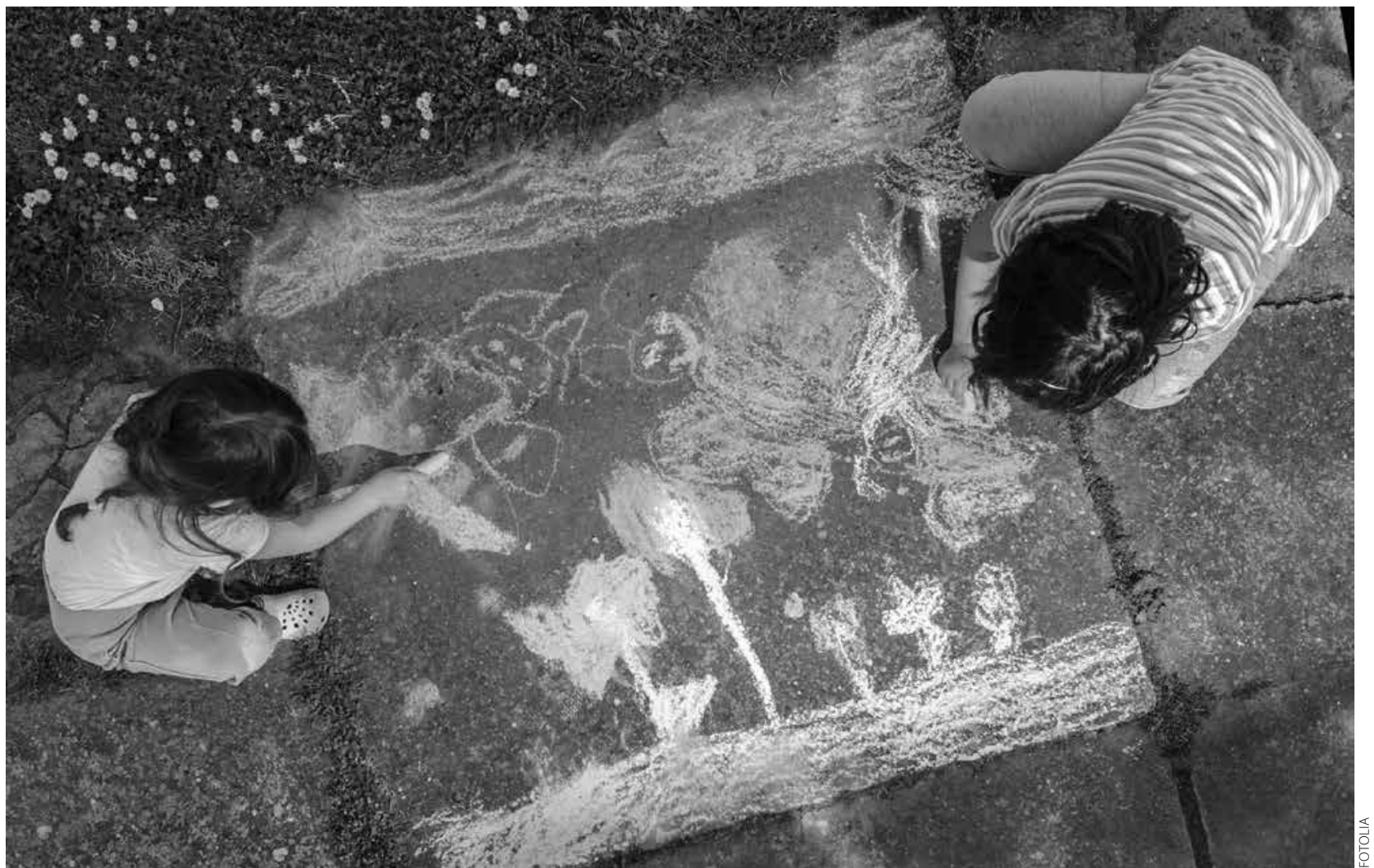

Les sorties en dehors du quartier : des déplacements domicile-école et des activités extrascolaires sous l'emprise de la mobilité résidentielle

Les enfants sans logement passent beaucoup de temps en dehors de leur quartier, en particulier dans les transports en commun. Une scolarisation extracommunale en est la principale explication : deux enfants scolarisés sur dix ne le sont pas dans une école de leur commune. Cette valeur est d'autant plus élevée pour les enfants qui déménagent : alors que près de neuf enfants stables sur dix sont inscrits dans leur commune, plus d'un quart des enfants ayant déménagé durant l'année fréquentent un établissement extracommunal. Ce découplage entre la commune de résidence et la localisation de l'école est associé à des retards en classe et à des difficultés d'apprentissage (Le Méner et Oppenchaim, 2015b). Mais ce n'est pas la seule conséquence de ce découplage sur les déplacements quotidiens des enfants.

De façon peu surprenante, le temps de trajet pour aller à l'école est corrélé à l'instabilité résidentielle : les enfants qui déménagent le moins mettent le moins de temps pour s'y rendre. Si $18 \%$ des enfants sans logement mettent plus d'une demi-heure pour aller à l'école, ceux qui n’ont pas déménagé sont $15 \%$ dans ce cas, contre $24 \%$ parmi ceux qui ont déménagé au moins une fois dans les douze derniers mois. L'éloignement entre l'école et l'hébergement se lit également dans l'utilisation plus fréquente des transports en commun, notamment du bus, par rapport aux enfants franciliens de leur âge (Driea, 2012). Pour aller à l'école, $23 \%$ des enfants sans logement empruntent le bus ( $5 \%$ en moyenne pour les enfants franciliens), $9 \%$ le métro $(0,8 \%$ pour les enfants franciliens) et $5 \%$ le RER ou le train $(0,4 \%$ pour les autres enfants). À l'inverse, l'usage de la voiture est quasi exceptionnel (il ne concerne que $2 \%$ d'entre eux, alors que près d'un quart des enfants franciliens y ont recours). Si la marche est la principale façon de se rendre à l'école (dans $67 \%$ des cas), elle concerne bien moins les enfants exposés à l'instabilité résidentielle, qui se déplacent avant tout en transport en commun : $75 \%$ des enfants n'ayant pas déménagé dans l'année se rendent à pied à l'école, $69 \%$ parmi ceux qui ont déménagé une fois et $32 \%$ parmi ceux qui ont déménagé plus d'une fois. Cela n'est pas sans incidence sur l'appréhension du trajet vers l'école : $75 \%$ des enfants n'ayant pas déménagé « adorent » ou «aiment bien » ces moments, contre $58 \%$ des enfants ayant déménagé. Ces longs trajets en transports en commun ne sont certes pas uniquement un temps perdu : un tiers des enfants en profitent pour jouer, $27 \%$ lisent, plus d'un quart écoutent de la musique, $13 \%$ font leurs devoirs ou dorment. Les enfants peuvent également y forger des compétences urbaines, notamment la capacité à se repérer dans le réseau de transports en commun : près des deux tiers des enfants connaissent ainsi au moins une ligne et une station s'y situant. 
Plus généralement l'instabilité résidentielle a également pour effet de limiter les activités extrascolaires des enfants en dehors de leur quartier. Si les enfants stables au niveau résidentiel ont logiquement moins tendance $(13 \%)$ que les autres $(22 \%)$ à quitter leur quartier depuis un mois pour aller à l'école, ils sont beaucoup plus nombreux à en être sortis pour réaliser une activité extrascolaire durant cette période $(73 \%$ contre $56 \%$ parmi les enfants ayant déménagé au moins une fois). Les enfants qui déménagent profitent moins que les autres des sorties scolaires : $7 \%$ d'entre eux y ont participé, contre plus d'un enfant stable sur cinq. De même, ils rendent moins visite aux membres de leur famille en dehors de leur quartier (une fois sur dix, soit trois fois moins que les enfants stables); ils fréquentent également moins souvent des magasins en dehors de leur quartier (dans $13 \%$ des cas, contre $28 \%$ chez les enfants n'ayant pas déménagé). Enfin, les enfants ayant déménagé partent moins souvent en vacances : si $33 \%$ des enfants sans logement sont partis lors des dernières grandes vacances ${ }^{6}$, ceux qui ont déménagé plus d'une fois ne sont que $16 \%$ dans ce cas. Cependant, cet effet de l'instabilité est beaucoup moins fort que celui de l'arrivée récente en France : seuls $4 \%$ des enfants dont les parents sont arrivés il y a moins de deux ans en France sont partis en vacances, contre $46 \%$ parmi ceux arrivés il y a plus de deux ans. Du reste, lorsqu'ils partent en vacances, ces enfants rendent moins souvent visite à des copains, à des membres de leur famille, ou à des amis de leurs parents. Par exemple, durant les dernières vacances, 58 \% des enfants stables résidentiellement ont vu des membres de leur famille en dehors de leur structure d'hébergement, alors que seul un quart des enfants ayant déménagé est dans ce cas.

Comment comprendre cette moindre mobilité des enfants instables en dehors de leur quartier ? L'instabilité résidentielle décroît avec le temps passé dans le système d'hébergement, qui est lui-même corrélé au temps passé en France. On peut penser que la période où l'instabilité est la plus importante correspond à une phase de découverte du territoire, sur laquelle retentit en retour la vulnérabilité résidentielle des familles et la longueur des déplacements domicile-école. Dans cette période, le monde des enfants pourrait être d'autant plus resserré sur l'école d'une part, et sur le foyer familial d'autre part. Il y aurait alors une franche limitation des activités enfantines en dehors de ces deux espaces. En contrepoint, la stabilisation résidentielle serait une condition de découverte et d'appropriation d'autres espaces, que ce soit le quartier ou des lieux plus éloignés.

6. En population générale, les trois quarts des enfants de cinq à dix-neuf ans partent au moins une fois par an en vacances (pour au moins quatre jours en dehors de leur domicile), d'après l'Enquête Vacances de l'Insee ; cette proportion passe à $50 \%$ chez les enfants dont la famille a un revenu mensuel inférieur à 1500 euros (Ovlej, 2013). Le Credoc estime que dans le premier décile des niveaux de vie, seuls $37 \%$ des ménages partent ainsi en vacances (Hoiban, 2012).
Les pratiques spatiales des enfants sans logement paraissent largement configurées par le type d'hébergement proposé et peut-être plus encore par leur mobilité résidentielle. Les différents établissements sont inégalement ouverts aux sociabilités enfantines. Cette inégale hospitalité renvoie à des modes de prise en charge et par là même à des services différents : tantôt l'hébergement répond à un ensemble de besoins, certains relatifs à la présence d'enfants ; tantôt, comme en hôtel social, il ne consiste qu'en une mise à l'abri, un hébergement « sec » comme l'analysent certains responsables de l'hébergement hôtelier. Dans ce dernier cas, la logique de l'action publique est profondément paradoxale. La protection qu'elle accorde à des étrangers, y compris sans-papiers, dépend de la présence d'enfants mineurs au sein des ménages ; celle-ci a longtemps valu comme un critère d'inconditionnalité effective de l'accueil. Mais l'organisation de l'hébergement traite les enfants comme n'importe quel adulte, sans préoccupation pour leur éducation ou pour leur développement et leur santé (Guyavarch et al., 2014). Dès lors, la vulnérabilité résidentielle qui touche les familles semble une autre traduction du règne du provisoire qui préside à l'hébergement des familles. Les simples descriptions statistiques présentées ici montrent bien l'effet corrosif de l'instabilité résidentielle sur les sociabilités et les pratiques enfantines, également remarqué dans des enquêtes menées auprès d'enfants sans logement, en Australie notamment (Moore et al., 2008 ; Kirkman et al., 2010). Tendanciellement, l'instabilité résidentielle paraît entrainer une désarticulation entre les espaces du quotidien, c'est-à-dire entre les activités, les relations sociales et les formes d'appropriation spatiales qui s'y font jour.

Dans d'autres milieux sociaux, un clivage des sociabilités enfantines a également pu être observé entre l'école, ses abords et les quartiers. Chez des enfants de milieu modeste, habitant dans des quartiers populaires mais fréquentant des écoles situées dans des quartiers gentrifiés, la mixité des relations sociales peut être réelle, mais aussi relativement limitée à l'espace scolaire et aux activités autour de l'école (Authier et LehmanFrisch, 2012). La socialisation familiale et le rapport au quartier des enfants rendent compte des formes plus ou moins prononcées de mixité sociale dans leurs pratiques quotidiennes, et de la possible dualité entre espace scolaire et espace d'habitation. Dans notre cas, les conditions d'hébergement et l'instabilité résidentielle révèlent d'autres clivages. Elles permettent alors d'expliquer qu'à une forte intégration au monde de l'école répond un resserrement des relations enfantines sur le foyer familial chez les enfants les plus vulnérables sur le plan résidentiel. 


\section{Références bibliographiques}

Authier J.-Y. (dir), (2010), État des lieux sur les trajectoires résidentielles, La Défense, Puca.

Authier J.-Y., Lehman-Frisch S., (2012), « Il était une fois... des enfants dans des quartiers gentrifiés à Paris et à San Francisco », Actes de la recherches en sciences sociales, $n^{\circ} 195$, pp. 58-73.

Buckner J.-C., (2008), « Understanding the impact of homelessness on children. Challenges and future research directions », American Behavioral Scientist, 51(6).

Cazottes E., (2013), «Question de la "pauvreté des enfants" en Europe. Nouvelles tendances dans les politiques publiques », Pensée plurielle, n 32 , pp. 95-113.

Charrier R. et al., (2009), Les conditions de logement en ile-de-France en 2006, Paris, IAU IDF/Insee/Dreif.

Chemineau D., Flamand J., (2014), « Les enfants de foyers allocataires à bas revenus en île-de-France. Évolution et caractéristiques de la pauvreté infantile de 2009 à 2013 », Dossier d'études des Caf en Île-deFrance, $n^{\circ} 3$

Choppin K., Gardella E. (dir.), (2012), Les sciences sociales et le sans-abrisme. Recension bibliographique de langue française, 1987-2012, Saint-Étienne, Publications de l'université de Saint-Étienne.

Donzeau N., Pan Ké Shon J.-L., (2009), « L'évolution de la mobilité résidentielle française de 1973 à 2006 », Population, 64(4), pp. 779-795.

Dourlens C., (2006), « L'accueil des demandeurs d'asile dans les structures d'urgence », Les Travaux de l'Observatoire 2005-2006, Paris, La Documentation française, pp. 687-715.

Driea, (2012), Enquête globale Transports, Stif/Driea/ Omnil/IAU IDF

Frigoli G., (2004), « Le demandeur d'asile : un "exclu" parmi d'autres ? La demande d'asile à l'épreuve des logiques de l'assistance », Revue européenne des migrations internationales, vol. 20, n² 2, pp. 153-167.
Guyavarch E., Garcin E., (2014), «Publics hébergés par le 115 de Paris : une forte progression des familles», Informations sociales, $n^{\circ} 182$, pp. 142-149.

Guyavarch E. et al., (2014), L'enquête Enfams (enfants et familles sans logement en Île-de-France). Premiers résultats, Rapport de l'Observatoire du Samusocial de Paris.

Guyavarch E., Le Méner E., (2014), « Les familles sans domicile à Paris et en Île-de-France : une population à découvrir », Politiques sociales et familiales, 115 , pp. 80-87.

Hoibian S., (2012), « Les catégories défavorisées, de plus en plus sur le bord de la route des vacances », Note de synthèse, Credoc, juillet.

Kirkman M. et al., (2010), «"Are we moving again this week?" Children's experiences of homelessness in Victoria, Australia », Social Science \& Medicine, vol. 70, n7, pp. 994-1001.

Labrador J., (2011), « Huit enfants de familles pauvres sur dix vivent dans un logement surpeuplé », Regards sur... les enfants de familles pauvres en île-de-France, Paris, Insee Île-de-France.

Le Méner E., (2013), «L'hôtellerie sociale : un nouveau marché de la misère ? Le cas de l'̂le-de-France », Politiques sociales et familiales, $n^{\circ} 114$, pp. 7-18

Le Méner E., (2015), «Vivre à l'hôtel quand on est un enfant », Métropolitiques, [en ligne] http://www. metropolitiques.eu/Vivre-a-I-hotel-quand-on-est-un. html, (mis en ligne le 17/04/2015).

Le Méner E., Oppenchaim N., (2012), «The temporary accommodation of homeless families in île-deFrance: between social emergency and immigration management », European Journal of Homelessness, vol. $6, n^{\circ} 1$, pp. 83-103.

Le Méner E., Oppenchaim N., (2015a), « Pouvoir aller à l'école. La vulnérabilité résidentielle d'enfants vivant en hôtel social », Les Annales de la recherche urbaine, $n^{\circ} 110$.
Le Méner E., Oppenchaim N., (2015b), «L'attachement à l'école des familles sans logement à l'épreuve de l'instabilité résidentielle », Métropolitiques, [en ligne] http://www.metropolitiques.eu/L-attachement-a-lecole-des.html, (mis en ligne le 15/06/2015).

Marpsat M., De Peretti G., (2009), « Une personne sur vingt s'est retrouvée sans logement personnel au cours de sa vie », Insee Première, 1255

Moore T., McArthur M., Noble-Carr D., (2008), "Stuff you'd never think of. Children talk about homelessness and how they'd like to be supported ", Family Matters, 78, pp. 36-43.

Mougin V., (2009), Papa, maman, la rue et moi. Quelle vie de famille pour les sans-domicile?, (photos de P. Bachelet), Paris, Éditions Pascal Bachelet.

Noblet P., (2001). «L'État, les associations d'accueil et d'hébergement et les étrangers demandeurs d'asile ou sans-papiers », Fondations.

Ovlej, (2013), « Le départ en vacances des enfants et des adolescents aujourd'hui : progression des inégalités et resserrement autour de la famille», Bulletin de l'Observatoire des vacances et des loisirs des enfants et des jeunes, $n^{\circ} 41$

Stettinger V., (2014). «Pour une approche sociologique renouvelée des enfants pauvres», Sociologie, $n^{\circ} 4$, vol. 5 .

Yaouancq F. et al., (2013), « L'hébergement des sans-domicile en 2012. Des modes d'hébergement différents selon les situations familiales », Insee Première, 1455

\section{Biographies}

EMMANUELLE GUYAVARCH est démographe. Elle a effectué sa thèse de doctorat au sein de l'Institut national d'études démographiques, puis a travaillé à I'Institut de recherche pour le développement et à I'Observatoire national de l'enfance en danger avant de rejoindre l'Observatoire du Samusocial de Paris en 2009, qu'elle dirige depuis 2010.

e.guyavarch@samusocial-75.fr
ERWAN LE MÉNER est sociologue, affilié au Centre d'étude des mouvements sociaux (Cems). II conduit sa thèse de doctorat sur l'hébergement d'urgence des familles. Il est également chercheur à I'Observatoire du Samusocial de Paris, où il mène des enquêtes concernant les populations sans logement.

e.lemener@samusocial-75.fr
NICOLAS OPPENCHAIM est maître de conférences en sociologie à l'université François Rabelais de Tours et chercheur au laboratoire Citeres (UMR 7324). II a étudié durant sa thèse de doctorat les pratiques de mobilité d'adolescents vivant dans des quartiers ségrégués et il a travaillé ensuite à l'Observatoire du Samusocial de Paris sur les enfants sans logement.

nicolas.oppenchaim@univ-tours.fr 